\title{
La SUVA se trompe de public cible
}

\section{Anna Sax}

Lic. oec. publ., MHA, membre de la rédaction

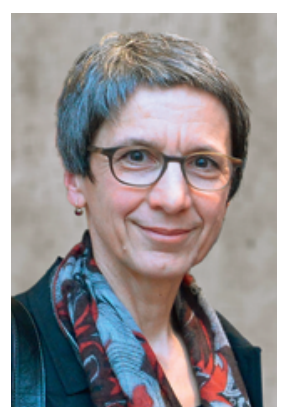

Tous les ans, entre 20 et 30 cyclistes, utilisateurs de vélos électriques compris, meurent dans un accident de la circulation, sans compter les 1000 blessés graves. D'après la statistique des accidents, alors que le nombre de blessés graves et de morts dans les accidents de voiture et de moto diminue continuellement, malgré l'intensification de la circulation, il reste constant pour les cyclistes et augmente même pour les utilisateurs de vélos électriques. A en croire les nombreux commentaires malveillants des médias, il faudrait en conclure que la responsabilité revient entièrement aux cyclistes mal élevés qui ne respectent pas les règles de la circulation (les «velorowdies»). C'est la faute des imbéciles. C'est effectivement un "con» que met en scène le clip actuel de la SUVA, censé favoriser la prévention des accidents. La vidéo présente les cyclistes comme de parfaits imbéciles. Elle montre un jeune père de famille, qui décède en allant travailler à vélo, car il n'a pas res-

Plus on roule entouré de deux tonnes de tôles, pour aller d'un point $\mathrm{A}$ à un point $\mathrm{B}$, moins on craint pour sa propre vie.

pecté les règles de circulation. «La mort [par contre], c'est rouler comme un con à vélo", explique une voix off après l'accident mortel. Et quand le collègue de travail constate que, pour une fois, c'est lui l'automobiliste qui arrive au bureau avant le cycliste, il commente avec cynisme: «Pfft... Il a encore dû crever.» Quant à moi, je suis restée sans voix quand j'ai vu le clip pour la première fois. Comment peut-on être aussi irrespectueux dans une campagne de prévention des accidents?

J'en suis alors venue à examiner de plus près la statistique des accidents. Elle indique que, globalement, la circulation routière est devenue plus sûre. Voilà déjà une bonne chose. Mais cet aspect positif de la sécurité routière est inégal. Elle s'est surtout améliorée pour les automobilistes, qui bénéficient de la protection de zones déformables et d'airbags de plus en plus efficaces. Grâce à ces dispositifs, en 2016 la Suisse a compté un quart de blessés graves et de morts de moins qu'en 2012, soit 947 au lieu de 1204. Même les motards ont circulé plus en sécurité en 2016 que les quatre années précédentes. Il n'y a que pour les cyclistes que le danger d'un accident aux conséquences graves n'a pas diminué. Mais c'est un peu simple de rejeter la responsabilité sur leur comportement imprudent et distrait.

Le clip de la SUVA nous dit que dans près de la moitié des accidents de vélo, le cycliste est responsable. Cette affirmation est à la fois vraie et fausse, car la statistique compte aussi ceux qui ont eu un accident tout seuls, n'impliquant aucun autre usager de la circulation. Mais quand les vélos entrent en collision avec des véhicules motorisés, comme dans l'accident mortel mis en scène dans la vidéo, les chiffres ne sont pas du tout les mêmes: en effet, dans près des trois quarts des cas, le conducteur automobile est le principal responsable de l'accident. Les cyclistes sont encore moins souvent à l'origine des collisions quand ces dernières se produisent en semaine, aux heures de pointe, entre 6 et 9 heures. La situation présentée dans la vidéo, dans laquelle un cycliste décède dans un choc qu'il a provoqué lui-même avec un automobiliste, est statistiquement très rare.

Il existe incontestablement des imbéciles parmi les cyclistes. Mais il n'est pas juste de rendre responsables tous les cyclistes victimes d'une collision avec un automobiliste, car ils ne le sont que dans un quart des cas. Les statistiques montrent en revanche que plus on roule entouré de deux tonnes de tôles, pour aller d'un point $\mathrm{A}$ à un point $\mathrm{B}$, moins on craint pour sa propre vie. C'est quand le trafic pendulaire est le plus fébrile que les cyclistes souffrent des préjudices les plus importants. Quand on est stressé et qu'on conduit nerveusement, on peut ne pas voir un cycliste. Les ronds-points sont particulièrement dangereux. Ces infrastructures très répandues, destinées à fluidifier la circulation des véhicules motorisés, sont de véritables pièges pour les vélos. Et, puisqu'on parle de règles de la circulation: je vois tous les jours des automobilistes qui les enfreignent, par exemple en coupant la route aux cyclistes qui sont sur la file de droite. La SUVA pourraitelle viser le bon public cible dans sa prochaine vidéo de prévention? 